\title{
Depressão como fator de risco para suicídio: Uma revisão sistemática
}

\author{
Depression as a risk factor for suicide: A systematic review \\ La depresión como factor de riesgo de suicidio: Una revisión sistemática
}

Recebido: 13/05/2021 | Revisado: 18/05/2021 | Aceito: 21/05/2021 | Publicado: 06/06/2021

\author{
Igor Mendes Furtado \\ ORCID: https://orcid.org/0000-0001-6494-7189 \\ Centro Universitário de Patos, Brasil \\ E-mail: mendesigor719@gmail.com \\ Osman Batista de Medeiros Filho \\ ORCID: https://orcid.org/0000-0002-4850-0667 \\ Centro Universitário de Patos, Brasil \\ E-mail: osmanbmf@ hotmail.com
}

\begin{abstract}
Resumo
Objetivo: Analisar a relação existente entre a depressão como fator de risco para o suicídio. Metodologia: Foi adotado o método de Revisão Sistemática da Literatura, cuja questão norteadora foi "Como a depressão influencia diretamente para o aumento das taxas de suicídio"? A temática da pesquisa foi buscada nas bases de dados Scientific Electronic Library Online (SCIELO) e na U. S. National Library of Medicine (PUBMED), através da implantação dos Descritores em Ciências da Saúde (DeCS): ("suicide” AND "depression"). Os critérios de inclusão utilizados foram: presença dos DeCS, idioma português ou espanhol, texto completo disponível, estudos em humanos e estudos transversais. Após a aplicação dos filtros, apenas sete artigos se encaixaram nos critérios de elegibilidade. Resultados: Foi evidenciado através dos autores que as taxas da prática suicida relacionados à depressão têm aumentado de forma significativa, configurando-se em uma das principais causas de morte e problemas de saúde pública enfrentados na atualidade. Conclusão: Através desse estudo, foi revelado que as taxas de práticas suicidas relacionados a transtornos depressivos são notoriamente elevadas e que é necessário a existência de ferramentas científicas e econômicas para haver a prevenção dos casos de suicídios na esfera global.
\end{abstract}

Palavras-chave: Suicídio; Depressão; Saúde mental.

\begin{abstract}
Objective: To analyze the relationship between depression as a risk factor for suicide. Methodology: The method of Systematic Literature Review was adopted, whose guiding question was "How does depression directly influence the increase in suicide rates"? The research theme was searched in the Scientific Electronic Library Online (SCIELO) and in the U. S. National Library of Medicine (PUBMED) databases, through the implementation of the Health Sciences Descriptors (DeCS): ("suicide" AND "depression"). The inclusion criteria used were: presence of the DeCS, Portuguese or Spanish language, full text available, studies in humans, and cross-sectional studies. After applying the filters, only seven articles met the eligibility criteria. Results: It was evidenced by the authors that the rates of suicidal practices related to depression have increased significantly, being configured as one of the main causes of death and public health problems faced today. Conclusion: Through this study, it was revealed that the rates of suicidal practices related to depressive disorders are notoriously high, and that the existence of scientific and economic tools is necessary for the prevention of suicide cases in the global sphere.
\end{abstract}

Keywords: Suicide; Depression; Mental health.

\section{Resumen}

Objetivo: Analizar la relación existente entre la depresión como factor de riesgo de suicidio. Metodología: Se adoptó el método de Revisión Sistemática de la Literatura, cuya pregunta guía fue "¿Cómo influye la depresión directamente en el aumento de las tasas de suicidio? El tema de la investigación se buscó en la Scientific Electronic Library Online (SCIELO) y en las bases de datos de la U. S. National Library of Medicine (PUBMED), mediante la aplicación de los Descriptores en Ciencias de la Salud (DeCS): ("suicidio" Y "depresión"). Los criterios de inclusión utilizados fueron: presencia del DeCS, idioma portugués o español, texto completo disponible, estudios en humanos y estudios transversales. Tras aplicar los filtros, sólo siete artículos cumplían los criterios de elegibilidad. Resultados: Se evidenció por parte de los autores que las tasas de práctica suicida relacionadas con la depresión han aumentado significativamente, configurándose como una de las principales causas de muerte y problemas de salud pública que se enfrentan en la actualidad. Conclusión: A través de este estudio, se puso de manifiesto que las tasas de prácticas suicidas relacionadas con los trastornos depresivos son notoriamente altas y que es necesaria la existencia de herramientas científicas y económicas para la prevención de los casos de suicidio en el ámbito mundial. 
Palabras clave: Suicidio; Depresión; Salud mental.

\section{Introdução}

Por definição, o suicídio é visto como qualquer ato de morte que é resultado, direta ou indiretamente, de um ato positivo ou negativo praticado pela própria vítima, onde esta por muitas vezes tem a consciência do resultado que irá produzir (Hernández, González, Ganen, Brito \& Macías, 2019). Por outro lado, a ideação suicida é descrita como o aparecimento de pensamentos em que o próprio indivíduo é a fonte da própria morte (Ranuzi, Santos, Araújo \& Rodrigues, 2020). Ademais, a tentativa do suicídio é qualquer ato através do qual o indivíduo fere a si mesmo, independentemente da forma utilizada para consumar o ato e o real motivo que a levou a praticar tal feito (Hernández, González, Ganen, Brito \& Macías, 2019). A idealização suicida e os cometimentos de suicídio fatal e não fatal geralmente iniciam aos 12-13 anos e tendem a aumentar durante a adolescência, e consequentemente podem estabelecer um determinado padrão de comportamento suicida em adultos jovens (Lejderman, Parisotto \& Spanemberg, 2020).

O comportamento suicida atualmente é visto como um dos principais problemas de saúde enfrentados pelos profissionais de saúde mental, à medida que com o passar dos anos aumenta o número de atos suicidas consumados. Muito embora a relação entre suicídio e transtornos mentais, principalmente a depressão, esteja bem evidenciada, outros fatores como o defrontamento de conflitos, violência, perdas familiares, isolamento e outras tentativas anteriores também se encontram fortemente ligados ao comportamento suicida (Ranuzi, Santos \& Araújo, 2020). O suicídio por vezes é visto como escolha para lidar com as dificuldades e algumas circunstâncias que a vida emprega, e esse fato tem acompanhado a história da humanidade e se demonstra de várias maneiras, desde a ideação suicida, até a própria consumação do ato (Hernández, González, Ganen, Brito \& Macías, 2019). É estimado que a depressão seja responsável por 4,3\% da totalidade mundial de todas as doenças e está entre as maiores causas de incapacidade no mundo, principalmente entre as mulheres, sendo a depressão caracterizada pela continuidade progressiva de sintomas depressivos associados a variação de humor repentino, que tem a capacidade de transformar a forma de ver o mundo e realidade transformada (Silva et al, 2015).

Até os dias atuais existem pessoas que enxergam o suicídio não como uma patologia psicológica, associando-o mais a fatores espirituais do que com os distúrbios mentais que um ser humano pode ser acometido. Então, é de suma importância que haja cada vez mais estudos que busquem o entendimento maior sobre as diversas patologias que afetam a mente humana, sendo uma das principais a depressão, que hoje é vista como um dos principais gatilhos que despertam o desejo suicida. A depressão está representada como o antecedente pessoal patológico mais presente entre as pessoas com comportamento suicida e, portanto, é o principal fator de risco encontrado nas tendências a prática suicidária (Hernández, González, Ganen, Brito \& Macías, 2019).

A depressão apresenta como principal característica a alteração de humor, que pode ser classificada em três graus, leve, moderado ou grave. Porém, existem características em comum que as pessoas afetadas com a depressão apresentam, que é a diminuição da capacidade de experimentar vivências que geram prazer, que em termos médicos é chamado de anedonia, a diminuição do nível de humor, a redução das práticas de atividades rotineiras, ideação de culpa ou indignação, insônia e perca de apetite (Sousa \& Tavares, 2020). No Brasil, entre os anos de 2011 a 2016, foram notificados 48.204 casos de tentativas de suicídio, estando dividido em $69 \%$ entre as mulheres e $31 \%$ entre os homens. Já o número de óbitos pela prática suicidária, nesse mesmo período atingiu o número de 55.649 casos (Meine, Cheiram \& Jaeger, 2019).

A perspectiva do suicídio é bastante delicada, tendo em vista que deve ser entendida como um conjunto de associações entre fatores psicológicos, comportamentais, sociais, culturais e de saúde que atuam simultaneamente, e principalmente por ser um tipo de morte que é considerada evitável. Nesse ponto de vista, o foco precisa ser o reconhecimento 
antecipado de sinais que o indivíduo demonstra e o consequente manejo adequado, que irá resultar em um importante alvo de prevenção ao comportamento suicidário (Ranuzi, Santos, Araújo e Rodrigues, 2020).

Portanto, é muito importante haver cada vez mais estudos que busquem expor as diversas causas que desencadeiam essa patologia que vem aumentando o número de óbitos a cada ano. Segundo a Organização Mundial de Saúde (OMS, 2013) cerca de 1.110 pessoas cometem suicídio por dia, estando entre as 10 principais causas de morte em todos os países, fazendo com que se emitam alertas constantes sobre o aumento dessas taxas (Trujillo, Elías \& Acosta, 2013). O suicídio é causador de aproximadamente 800.000 mortes globais anualmente, sendo que nestes números não estão incluídos os números de tentativas de suicídio, os quais estudos comprovam que ocorrem em torno de 10 a 20 vezes a mais do que o próprio ato consumado. Em dados gerais, o nível global da prática suicidária é de 10,6 a cada 100.000 habitantes, o que corresponde a 1,4\% de todas as mortes em todo o mundo. No Brasil, o índice de mortalidade decorrente do suicídio está em torno de 6,1 por 100 mil habitantes, estando entre os dez países com maior número de suicídios (Freire et al, 2020). A identificação precoce de pessoas que apresentam risco de praticar a atividade suicidária é essencial para garantir o melhor atendimento, tendo em vista que os médicos de família desempenham um papel importante para essa identificação, pois a maioria dos indivíduos que perderam a vida por suicídio foi a consulta no posto de saúde no mês anterior a morte e cerca de $90 \%$ consultou o médico de família várias vezes durante o ano anterior ao ato suicida (Martinengo, Galen, Lum, Kowalski, Subramaniam \& Car, 2019).

Além disso, é de muita valia obter o conhecimento suficiente para se alcançar um tratamento efetivo para essas psicopatologias. É necessário que haja um manejo multiprofissional, principalmente entre os psiquiatras e psicólogos.

O objetivo principal desse estudo é buscar o entendimento sobre a relação existente entre os distúrbios que provocam a prática suicida, principalmente a depressão, e o consequente aumento das taxas dessa prática a cada ano.

\section{Metodologia}

O presente estudo foi elaborado a partir do método de Revisão Sistemática da Literatura, que é agrupado dentro dos tipos secundários, tendo em vista que fazem uso dos estudos primários como principais fontes de dados (Galvão \& Pereira, 2014). Além disso, esta forma de pesquisa proporciona uma síntese dos indícios que se relaciona com um plano de intervenção, por meio da utilização de métodos explícitos e sistemáticos de busca, observação, crítica e construção da informação selecionada (Sampaio \& Mancini, 2007).

A composição da Revisão Sistemática da Literatura, de acordo com Galvão, Sawada e Trevizan (2004), compreende o preenchimento de sete fases, sendo elas: construção do protocolo, definição da pergunta, busca dos estudos, seleção dos estudos, avaliação crítica dos estudos, coleta dos dados e síntese dos dados. Logo, a partir das fases descritas, foi empregada a estratégia PICO, que possui a função de estabelecer a questão norteadora, em que (P) representa a população, (I) simetriza a intervenção de interesse, (C) iguala-se ao controle ou comparação, e por fim, o (O) corresponde ao desfecho. A população (P) de interesse foram as pessoas que cometem suicídio e possuem depressão e o desfecho $(\mathrm{O})$ foi o próprio suicídio. Nesse tipo de revisão sistemática de taxa ou frequência, não se faz uso da intervenção (I) nem do comparador (C).

Portanto, a pergunta que foi elaborada se resume a: Qual a prevalência do suicídio relacionado às pessoas que possuem depressão? Esse tipo de pergunta norteadora é bastante relevante para evolução da revisão sistemática, tendo em vista que ela possibilitou o reconhecimento dos estudos e consequentemente quais foram os dados que necessitaram ser coletados em cada estudo.

Então, para a elaboração e seleção dos estudos, coleta e síntese de dados, buscou-se a temática da pesquisa nas bases de dados Scientific Electronic Library Online (SCIELO) e na U. S. National Library of Medicine (PUBMED), através da implantação dos Descritores em Ciências da Saúde (DeCS): (“suicide” AND “depression”), sendo encontrados um total de 429 
artigos na SCIELO e 1359 publicações na PUBMED. Os critérios de inclusão utilizados foram: presença dos DeCS, idioma português ou espanhol, texto completo disponível, estudos em humanos e estudos transversais. Após a aplicação dos filtros, apenas sete artigos se encaixaram nos critérios de elegibilidade.

Fluxograma 1 - Esquema de seleção dos estudos para a revisão sistemática pelo método PRISMA.

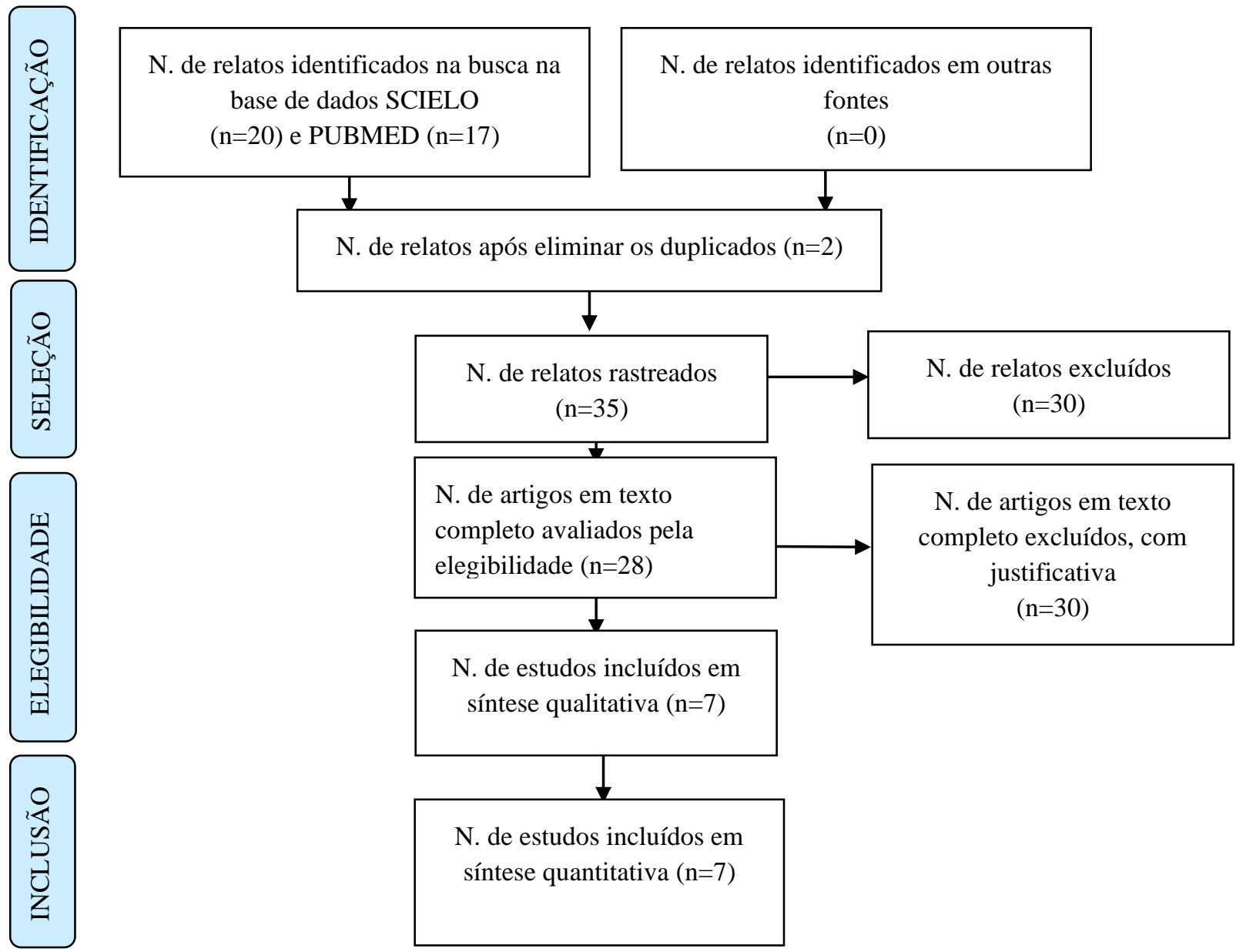

Fonte: Autores.

De acordo com os estudos selecionados, foi determinado os níveis de evidência classificando os artigos em quatro níveis distintos: alto, moderado, baixo e muito baixo. Foram utilizadas as seguintes informações: número de entrevistados em cada pesquisa, achados consistentes, tipo de estudo, a delimitação e a confiança que cada artigo fornece de acordo com seu objetivo estimado. Sendo assim, a partir desses critérios, 2 artigos foram classificados como nível alto, 2 artigos foram incluídos no nível moderado e 3 artigos preencheram o nível baixo dos 7 artigos no total. Destarte, foi empregado as Diretrizes Metodológicas to Sistema GRADE (BRASIL, 2014), que classifica a qualidade da evidência em:

Alto: Engloba os artigos onde há uma forte confiança de que o seu efeito esteja o mais próximo do estimado possível. Estão incluídos nesse nível os estudos observacionais ou os ensaios clínicos bem delineados, com amostra representativa.

Moderado: Compreende os artigos onde existe uma confiança moderada no efeito estimado, suscetível a modificações por trabalhos futuros. Estão englobados nesse nível os ensaios clínicos com leves limitações, além de estudos observacionais bem delineados com achados consistentes. 
Baixo: Abrange os artigos que possuem uma confiança limitada em seu efeito estimado, em que os trabalhos que surgirão futuramente terão uma importante repercussão. Fazem parte desse nível os ensaios clínicos com

?. moderadas limitações, além de estudos observacionais comparativos, como os de coorte e caso-controle.

Muito Baixo: Compreende os artigos que possuem um grau significativo de incerteza em seus achados, onde qualquer estimativa de efeito é incerta. Estão incluídos nesse nível os ensaios clínicos com graves limitações, opinião de especialistas e estudos observacionais não comparados e os comparativos com presença de limitações.

\section{Resultados}

De acordo com o quadro 1, foi visto que as publicações se encontram inseridas nas bases de dados PUBMED, sendo 4 disponíveis nessa base de dados, e na SCIELO, estavam 3 disponíveis. Onde 6 foram categorizados em Estudo Transversal e apenas 1 como Estudo de Coorte.

Quadro 1 - Descrição quanto a autores, ano, título, base de dados e tipo de estudo.

\begin{tabular}{|c|c|c|c|c|}
\hline Autor/Ano & Título & Base de dados & Tipo de estudo & Nível de evidência \\
\hline $\begin{array}{l}\text { Nascimento, V. et } \\
\text { al. (2019) }\end{array}$ & $\begin{array}{l}\text { Association between eating disorders, } \\
\text { suicide and depressive symptoms in } \\
\text { undergraduate students of health-related } \\
\text { courses. }\end{array}$ & PUBMED & Transversal & Moderado \\
\hline $\begin{array}{l}\text { Faure, M. I. et al. } \\
\text { (2018) }\end{array}$ & $\begin{array}{l}\text { Association of health-related quality of } \\
\text { life and suicidal risk in adolescents: A } \\
\text { cross-sectional study. }\end{array}$ & PUBMED & Transversal & Baixo \\
\hline $\begin{array}{l}\text { Freire, F.O. et al.. } \\
(2020)\end{array}$ & $\begin{array}{l}\text { Factors associated with suicide risk } \\
\text { among nurses and physicians: a cross- } \\
\text { section study. }\end{array}$ & SCIELO & Transversal & Moderado \\
\hline $\begin{array}{l}\text { Salvo, L., Ramírez, } \\
\text { J., \& Castro, A. } \\
(2019)\end{array}$ & $\begin{array}{l}\text { Risk factors for suicide attempts in } \\
\text { people with depressive disorders treated } \\
\text { in secondary health care. }\end{array}$ & PUBMED & Transversal & Baixo \\
\hline $\begin{array}{l}\text { Ato, R.S., Estrada, } \\
\text { M.A.V., Mejia, C., } \\
\text { Alvarado J.C. } \\
(2018)\end{array}$ & $\begin{array}{l}\text { Suicide risk associated with bullying and } \\
\text { depression in high school. }\end{array}$ & SCIELO & Transversal & Alto \\
\hline $\begin{array}{lr}\text { Ranuzi, C., } & \text { Santos, } \\
\text { T.G., } & \text { Araujo, } \\
\text { A.C.M.C., } & \& \\
\text { Rodrigues, } & \text { L.R. } \\
(2020) & \end{array}$ & $\begin{array}{l}\text { Suicidal thinking, } \\
\begin{array}{l}\text { depression, and } \\
\text { religiosity in a freedom-deprived } \\
\text { population. }\end{array}\end{array}$ & PUBMED & Transversal & Alto \\
\hline $\begin{array}{l}\text { Orellana, J.D.Y. et } \\
\text { al. (2020) }\end{array}$ & $\begin{array}{l}\text { Mental disorders in adolescents, young } \\
\text { people and adults from the consortium of } \\
\text { brazilian birth cohorts rps. }\end{array}$ & SCIELO & Coorte & Baixo \\
\hline
\end{tabular}

Fonte: Autores. 
De acordo com os autores dos artigos estudados, em diversos grupos de pessoas, as taxas de tentativas de suicídio entre estudantes segundo Nascimento et al. 2019 é de aproximadamente 17,3\% e desses alunos 13,6\% apresentaram sintomas de depressão grave e estavam em risco de suicídio. Em um estudo realizado por Faure et al. 2018 revelou que entre 128 adolescentes 16,4\% destes apresentaram risco para cometer suicídio. Por fim, Freire et al. 2020 constatou que a prevalência de suicídio ao longo da vida entre enfermeiros foi de 9,41\% e médicos de 2,29\% e ainda no mesmo artigo foi identificado um risco de $15,74 \%$ da prática suicida em relação à população em geral de profissionais da saúde de outros países. Portanto, foi verificado que a menor taxa de suicídio é de 2,29\% e a maior taxa apresentada é de 23,61\%, em grupos distintos de pessoas. Estima-se que o índice mundial de suicídio é de 16 para cada 100 mil habitantes, que varia de acordo com o sexo, idade e país e somente nos últimos 45 anos, essas taxas de suicídio tiveram um aumento de $60 \%$ em todo o mundo (Braga \& Dell'aglio, 2013).

Quadro 2 - Principais informações citadas pelos autores.

\begin{tabular}{|c|c|c|c|}
\hline Autor/Ano & Frequência/Taxa & Fatores Relacionados & Implicações \\
\hline $\begin{array}{l}\text { Nascimento, V. et } \quad \text { al. } \\
(2019)\end{array}$ & $\begin{array}{l}\text { Foi realizado um estudo transversal com } \\
271 \text { alunos e aproximadamente } 17,3 \% \text { dos } \\
\text { alunos apresentaram sintomas de depressão } \\
\text { grave e } 13,6 \% \text { estavam em risco de } \\
\text { suicídio, sendo dividido como sendo } 7,4 \% \\
\text { risco baixo, } 0,7 \% \text { risco moderado e } 5,5 \% \\
\text { como sendo risco alto de suicídio. }\end{array}$ & $\begin{array}{l}\text { Transtornos alimentares e } \\
\text { bulimia; } \\
\text { Sintomas de depressão grave; } \\
\text { Sexo feminino. }\end{array}$ & $\begin{array}{l}\text { Altas taxas de mortalidade e } \\
\text { incapacidade; } \\
\text { Diversas patologias físicas e } \\
\text { psicológicas; } \\
\text { Qualidade de vida } \\
\text { prejudicada. }\end{array}$ \\
\hline Faure, M. I. et al. (2018) & $\begin{array}{l}\text { Foi feito um estudo transversal com } 128 \\
\text { adolescentes entre } 15 \text { e } 19 \text { anos de Puerto } \\
\text { Aysen (Chile), os quais responderam a } \\
\text { entrevista EQ-5D-5L, de acordo com a } \\
\text { Escala de Suicídio Okasha, junto a duas } \\
\text { questões relacionadas ao risco iminente de } \\
\text { suicídio. Dentre estes adolescentes, } 16,4 \% \\
\text { apresentaram risco de praticar o suicídio. }\end{array}$ & $\begin{array}{l}\text { Depressão; } \\
\text { Tentativas anteriores de } \\
\text { suicídio; } \\
\text { Alterações psicológicas e } \\
\text { comportamentais } \\
\text { adolescentes; } \\
\text { Doenças crônicas em idosos; } \\
\text { Dinâmica familiar. }\end{array}$ & $\begin{array}{l}\text { Dor/desconforto } \\
\text { relacionados com a saúde } \\
\text { física; } \\
\text { Isolamento social e } \\
\text { familiar; } \\
\text { Consumo abusivo de álcool. }\end{array}$ \\
\hline Freire, F.O. et al. (2020) & $\begin{array}{l}\text { Estudo transversal executado em um } \\
\text { hospital universitário, onde } 216 \\
\text { trabalhadores da área de saúde foram } \\
\text { entrevistados e responderam ao } \\
\text { questionário sociodemográfico-laboral e o } \\
\text { Mini International Neuropsychiatric } \\
\text { Interview (MINI), que avaliou o risco de } \\
\text { suicídio e depressão. A prevalência de } \\
\text { tentativas de suicídio ao longo da vida } \\
\text { entre enfermeiras e médicos foram de } \\
9,41 \% \text { e } 2,29 \% \text { respectivamente. }\end{array}$ & $\begin{array}{l}\text { Não possuir } \\
\text { conjugal fixo; } \\
\text { História de } \\
\text { suicídio; }\end{array}$ & $\begin{array}{l}\text { Diminuição do rendimento } \\
\text { no trabalho; } \\
\text { Dificuldade nas relações } \\
\text { interpessoais; } \\
\text { Insônia; } \\
\text { Diminuição da qualidade de } \\
\text { vida. }\end{array}$ \\
\hline $\begin{array}{l}\text { Salvo, L., Ramírez, J., \& } \\
\text { Castro, A. (2019) }\end{array}$ & $\begin{array}{l}\text { Foi realizado um estudo com } 112 \text { pacientes } \\
\text { ambulatoriais em três centros de atenção } \\
\text { psiquiátrica de Nuble (Chile), onde foi } \\
\text { constatado que } 67 \% \text { dos entrevistados } \\
\text { possuíam ideação suicida e } 43,8 \% \\
\text { chegaram ao ato consumado do suicídio. } \\
\text { Foi visto ainda que o risco da atividade } \\
\text { suicidária foi maior em pessoas que }\end{array}$ & $\begin{array}{l}\text { Idade mais jovem associado ao } \\
\text { estado civil solteiro; } \\
\text { Sintomas psicóticos graves, } \\
\text { dentre eles a depressão; } \\
\text { Transtornos de personalidade; } \\
\text { Sexo feminino. }\end{array}$ & $\begin{array}{l}\text { Aumento no uso de } \\
\text { substâncias ilícitas; } \\
\text { Estresse pós traumático; } \\
\text { Impulsividade; } \\
\text { Desesperança. }\end{array}$ \\
\hline
\end{tabular}




\begin{tabular}{|c|c|c|c|}
\hline & possuem sintomas depressivos maiores. & & \\
\hline $\begin{array}{l}\text { Ato, R.S., Estrada, } \\
\text { M.A.V., Mejia, C., } \\
\text { Alvarado J.C. (2018) }\end{array}$ & $\begin{array}{l}\text { Foi desempenhado um estudo transversal } \\
\text { analítico em uma cidade no norte do Peru } \\
\text { com } 289 \text { escolares. Dos entrevistados, } \\
20,1 \% \text { apresentaram risco de suicídio e } \\
\text { algum tipo de depressão. Foi evidenciado } \\
\text { ainda que } 38 \% \text { dos estudantes } \\
\text { apresentaram ideação suicida relacionado à } \\
\text { prática do bullying. }\end{array}$ & $\begin{array}{l}\text { Bullying; } \\
\text { Transtorno depressivo; } \\
\text { Distúrbio leve de humor; }\end{array}$ & $\begin{array}{l}\text { Déficit de aprendizagem } \\
\text { escolar; } \\
\text { Isolamento e dificuldade de } \\
\text { relações interpessoais; } \\
\text { Uso precoce de álcool e } \\
\text { outras substâncias ilícitas; } \\
\text { Transtornos bipolares. }\end{array}$ \\
\hline $\begin{array}{l}\text { Ranuzi, C., Santos, T.G., } \\
\text { Araújo, A.C.M.C., \& } \\
\text { Rodrigues, L.R. (2020) }\end{array}$ & $\begin{array}{l}\text { Estudo transversal com perspectiva } \\
\text { quantitativa, abordando } 228 \text { participantes, } \\
\text { através de um questionário } \\
\text { sociodemográfico, dentro do contexto } \\
\text { privação de liberdade e sobre a presença de } \\
\text { pensamento suicida. A taxa encontrada de } \\
\text { pessoas com ideação suicida foi de } 23,61 \% \\
\text { durante o encarceramento. }\end{array}$ & $\begin{array}{l}\text { Falta de liberdade ou } \\
\text { encarceramento; } \\
\text { Transtornos depressivos; } \\
\text { Presença de pensamentos } \\
\text { suicidas anteriores; } \\
\text { Presença de sintomatologia de } \\
\text { estresse/ansiedade. }\end{array}$ & $\begin{array}{l}\text { Isolamento social; } \\
\text { Qualidade de vida } \\
\text { prejudicada; } \\
\text { Falta de ânimo e esperança. }\end{array}$ \\
\hline $\begin{array}{l}\text { Orellana, J.D.Y. et al. } \\
(2020)\end{array}$ & $\begin{array}{l}\text { Foi realizado estudo de Coorte na América } \\
\text { do Sul, onde constatou-se que a prevalência } \\
\text { de transtornos comportamentais com } \\
\text { ideação suicida nos últimos } 12 \text { meses foi de } \\
17 \%\end{array}$ & $\begin{array}{l}\text { Doenças neuropsiquiátricas; } \\
\text { Depressão e Ansiedade; } \\
\text { Sexo feminino; } \\
\text { Menor nível socioeconômico. }\end{array}$ & $\begin{array}{l}\text { Fobia social; } \\
\text { Violência urbana. }\end{array}$ \\
\hline
\end{tabular}

Fonte: Autores.

\section{Discussão}

É notório que ao longo dos anos as taxas de práticas suicidas tem aumentado de uma forma bem expressiva e motivados por diversos tipos de gatilhos e patologias, porém, a depressão aparece relacionada à grande maioria das causas de suicídio e isso revela que uma das principais ferramentas para a prevenção e consequente diminuição dos números de atividades suicidárias, seria tratar as causas que levam as pessoas a desenvolver a depressão, existe um determinado padrão entre as pessoas que possuem o desejo de praticar o suicídio. Dentre eles, os transtornos depressivos, os conflitos familiares, nível socioeconômico baixo, fobia social e doenças neuropsiquiátricas são os principais fatores relacionados com o desenvolvimento da prática suicida (Faure et al, 2018). Ainda nesse meio, é revelado que o suicídio está diretamente ligado a processos patológicos internos inerente ao próprio indivíduo e que devido a esse fator é necessário que haja intervenção e tratamento especializado através dos profissionais da saúde mental (Araújo, Coelho \& Lopes, 2016). Por ser o suicídio um fenômeno complexo, que abrange diferentes áreas da vida do sujeito por meio da psicologia, biologia, cultura, existência e entre outros aspectos, destaca-se a relação entre $90 \%$ dos casos a transtornos mentais, sendo a doença mental mais comum, a depressão (Souza \& Moreira, 2018). Diante disso, foi visto que a jovem idade, alterações de humor, histórico de abuso na infância, história de tentativa de suicídio associado a probabilidade e tentativa real, foram fatores prenunciadores estatisticamente significativos para a consumação do ato suicida (Melhem et al, 2019).

De um modo particular, de acordo com Nascimento et al. (2019), que realizou um estudo transversal com amostra de 271 alunos, aproximadamente 17,3\% dos alunos apresentaram sintomas de depressão grave e 13,6\% estavam em risco de suicídio, estando relacionado às alterações no estilo alimentar e à realização do controle de peso corporal, que estão diretamente ligados à insatisfação pessoal que ocasiona a baixa autoestima, o que leva as pessoas a tomarem atitudes que refletem de um modo negativo a saúde corporal e mental, como práticas exacerbadas de exercícios físicos, dietas alimentares 
restritivas, tudo voltado para obtenção de um corpo ideal que é empregado por meio das mídias sociais. Então, a partir desses fatos, a crescente prática do suicídio relacionado com a insatisfação corporal, nos últimos anos, levou à inclusão dos transtornos alimentares nas patologias neuropsiquiátricas. Dentre elas, a principal seria o desenvolvimento da própria depressão, por conta da busca infindável do corpo perfeito.

Segundo Faure et al. (2018), a partir de um estudo transversal com 128 adolescentes entre 15 e 19 anos de Puerto Aysen (Chile), foi evidenciado que 16,4\% apresentaram risco de praticar o suicídio, e as maiores taxas estão localizadas na região Sul do Chile, onde segundo projeções poderá aumentar em 39\% o número de casos de suicídio, se nenhuma medida preventiva for tomada. Portanto, o conhecimento dos fatores de risco e a aplicação de ferramentas econômicas para busca dos riscos são de suma importância para prevenção e criação de intervenções específicas. A depressão associada a tentativas anteriores de suicídio são os principais fatores associados à atividade suicidária, ainda que existem evidências que mostram que há diferenças nos perfis de riscos entre as diferentes faixas etárias, sendo os principais fatores entre adolescentes: as lesões autoinfligidas, a depressão, o consumo de álcool, a dinâmica familiar e as alterações comportamentais/psicológicas. Entre os adultos e idosos, as doenças crônicas e a diminuição da qualidade de vida são os fatores comumente encontrados para a prática suicida.

A partir de um estudo transversal, Freire et al. (2020) realizou em um hospital universitário uma entrevista com 216 trabalhadores da área de saúde que responderam ao questionário sociodemográfico-laboral e ao Mini International Neuropsychiatric Interview (MINI), que mensurou o risco de suicídio e depressão entre médicos e enfermeiros. Neste estudo, foi constatado que a prevalência de tentativas de suicídio ao longo da vida entre enfermeiras e médicos foram de $9,41 \%$ e $2,29 \%$, respectivamente. No que diz respeito aos principais fatores associados às causas da prática suicida, não possuir um companheiro conjugal representa um significativo fator de risco ao suicídio. O histórico de tentativas de suicídio representa um dos mais importantes preditores para a prática do suicídio completo, com evidências mostrando que as tentativas de suicídios tendem a ser recorrentes e aumentam em até cinco vezes mais o risco de tentar novamente a prática suicidária. Já em relação às pessoas que vivem em sofrimento mental constante e que apresentam algum tipo de transtorno depressivo, o estudo revela que esses fatores também apresentam grande significância nos números de casos de suicídios entre esses grupos de profissionais.

Salvo, Ramirez \& Castro (2019) mostraram através de um estudo transversal que a depressão é vista como o transtorno psiquiátrico mais comum em pessoas com comportamento suicida, através de um estudo de acompanhamento em 12 meses que foi feito com 112 pacientes ambulatoriais em três centros de atenção psiquiátrica de Ñuble, Chile, com avaliações iniciais e trimestrais. Através deste estudo, foi constatado que $67 \%$ dos participantes possuíam algum tipo de ideação suicida, e destes, 43,8\% tentaram suicídio. Foi visto ainda que o risco para a tentativa do suicídio foi notoriamente maior em pessoas que possuem algum tipo de transtorno depressivo maior. Os principais fatores de risco para a tentativa da prática suicidária foram a idade jovem, não possuir um companheiro conjugal fixo, o desemprego e possuir algum tipo de transtorno de personalidade. Um estudo desse tipo realizado no Chile mostrou que 76,7\% das pessoas que se suicidaram possuíam um episódio depressivo maior (EDM) no momento de sua morte. Da mesma forma, em pacientes deprimidos, são retratadas altas taxas de tentativas de suicídio e ideias suicidas de gravidade variada. (Salvo, Ramirez \& Castro, 2019).

Outro estudo transversal analítico, realizado por Ato, Estrada, Mejia e Alvarado (2018), em uma cidade no norte do Peru, demonstrou que entre 289 escolares, $20 \%$ mostravam risco de suicídio e demonstraram algum tipo de transtorno depressivo. Entre esses, 38\% dos alunos que sofriam bullying tiveram ideação suicida e os que se sentiam deprimidos, 63\% sentiam a mesma ideação da prática do suicídio. No estudo, também foi ressaltado que tentativas anteriores de suicídio representam um importante preditor do comportamento suicida e que haver estudos que verifiquem os fenômenos que afetam a saúde mental dessas pessoas é de fundamental importância. 
Segundo Ranuzi, Santos, Araújo e Rodrigues (2020), que realizou um estudo transversal através da abordagem quantitativa com 228 participantes em contexto da privação da liberdade, a partir de um questionário sociodemográfico, avaliou-se a presença do pensamento suicida. Foi evidenciado através de um cálculo amostral que dentre os entrevistados, 23,7\% apresentaram ideação suicida durante o encarceramento. Sendo assim, supõe-se que a privação da liberdade aumente a predisposição do indivíduo em desenvolver a ideação suicida.

Finalmente, através de um estudo de Coorte, realizado por Orellana et al. (2020), foi constatado que a prevalência de transtornos comportamentais com ideação suicida, relacionados à depressão, fobia social e sexo feminino, nos últimos 12 meses, foi de $17 \%$. Foram estudadas cinco coortes de nascimento, em diferentes faixas etárias, sendo duas em Ribeirão Preto, duas em Pelotas e uma em São Luís. Ao todo, 12.350 indivíduos foram incluídos nas análises.

\section{Conclusão}

O presente estudo revelou que as taxas de práticas suicidas relacionados a transtornos depressivos são notoriamente elevadas e, além disso, evidenciou as diversas psicopatologias que também promovem a ideação da atividade suicidária, havendo uma significativa importância para a busca de estudos que promovam a descoberta precoce dos principais fatores de risco para a ideação suicida e que permitam intervir e evitar a consumação do ato suicida.

Portanto, é de grande relevância haver estudos que busquem o maior entendimento sobre as afecções mentais que levam uma pessoa a cometer o suicídio, e como sugestão de um tipo de estudo, seria algo relacionado as taxas específicas de cada psicopatologia que promovem as atividades suicidárias, estratificando minunciosamente cada tipo de enfermidade psicológica em porcentagens, que permitiria haver uma intervenção mais efetiva de acordo com cada tipo de distúrbio.

\section{Referências}

Araújo, R., Coelho, Z. P., Felisbela L. F. (2016). Representações do suicídio na imprensa generalista portuguesa. Revista Portuguesa de Saúde Pública, 34(2), 173-185.

Ato, R.S., Estrada, M. A. V., Mejia, C., Alvarado J.C. (2018). Suicide risk associated with bullying and depression in high school. Revista chilena de pediatría, 89(2), 208-215.

Braga, L. L., \& Aglio, D. D. D. (2013). Suicídio na adolescência: fatores de risco, depressão e gênero. Contextos Clínicos, 6(1), 2-14.

Freire, F. O et al. (2020). Factores associados al riesgo de suicido entre enfermeiros y médicos: un estúdio transversal. Rev. Bras. A enferm. 73, supl. 1, (e20200352).

Brasil. (2014). Ministério da Saúde. Secretaria de Ciência, Tecnologia e Insumos Estratégicos. Departamento de Ciência e Tecnologia. Diretrizes metodológicas: Sistema GRADE - Manual de graduação da qualidade da evidência e força de recomendação para tomada de decisão em saúde. Brasília: Ministério da Saúde.

Faure, M. I. et al. (2018). Asociación entre la calidad de vida relacionada con la salud y riesgo suicida en adolescentes: estudio transversal [Association of health-related quality of life and suicidal risk in adolescents: A cross-sectional study]. Revista chilena de pediatria, 89(3), 318-324.

Freire, F.O et al. (2020). Factores associados al riesgo de suicido entre enfermeiros y médicos: un estúdio transversal. Rev. Bras. A enferm. 73, supl. 1, (e20200352).

Galvao, C. M., Sawada, N. O., \& Trevizan, M. A. (2004) Revisão sistemática: recurso que proporciona a incorporação das evidências na prática da enfermagem. Rev. Latino-Am. Enfermagem, 12(3), 549-556.

Galvão, T. F., \& Pereira, M. G. (2014) Revisões sistemáticas da literatura: passos para sua elaboração. Epidemiol. Serv. Saúde. 23(1), 183-184.

Hernández, G. L. R., González, A. M. V., Ganen, M. S., Brito M. G., \& Macías Y. C. (2019). Psicodinámica familiar y apoyo social en adolescentes con conducta suicida. Área VI. Cienfuegos. MediSur, 17(2), 191-200.

Lejderman, B., Parisotto, A., \& Spanemberg, L. (2020). Trends in suicidal behavior at a general hospital emergency department in southern Brazil. Trends in Psychiatry and Psychotherapy, 42(4), 311-317.

Martinengo, L., Galen, V.L., Lum, E., Kowalski M., Subramaniam M., \& Car, J. (2019) Suicide prevention and depression apps' suicide risk assessment and management: a systematic assessment of adherence to clinical guidelines. BMC Med 17, 23. 
Research, Society and Development, v. 10, n. 6, e46410616046, 2021

(CC BY 4.0) | ISSN 2525-3409 | DOI: http://dx.doi.org/10.33448/rsd-v10i6.16046

Meine, I. R., Cheiram, M. C., \& Jaeger, F. P. (2019). Depressão e suicídio: o adolescente frente a fatores de risco socioculturais. Research, Society and Developmen. 8(12) (e448121882).

Melhem, N. M. et al. (2019). Severity and Variability of Depression Symptoms Predicting Suicide Attempt in High-Risk Individuals. JAMA Psychiatry. 76(6), 603-613.

Nascimento, V. et al. (2019). Association between eating disorders, suicide and depressive symptoms in undergraduate students of health-related courses. Einstein (Sao Paulo, Brazil), 18 (eAO4908).

Oliveira, F.F. et al. (2020). Factors associated with suicide risk among nurses and physicians: a cross-section study. Revista Brasileira de Enfermagem, 73 (e20200352).

Orellana, J. D. Y. et al. (2021) Transtornos mentais em adolescentes, jovens e adultos do Consórcio de Coortes de Nascimento brasileiras RPS (Ribeirão Preto, Pelotas e São Luís). Cadernos de Saúde Pública, 36(2) (e00154319).

Ranuzi, C., Santos, T. G., Araujo, A. C. M. C., \& Rodrigues, L. R. (2020). Suicidal thinking, depression, and religiosity in a freedom-deprived population. Rev. Latino-Am. Enfermagem, 28 (e3368).

Salvo, L., Ramírez, J., \& Castro, A. (2019). Factores de riesgo para intento de suicidio en personas con trastorno depresivo en atención secundaria [Risk factors for suicide attempts in people with depressive disorders treated in secondary health care]. Revista medica de Chile, 147(2), 181-189.

Sampaio, R. F., \& Mancini, M. C. (2007) Estudos de revisão sistemática: um guia para síntese criteriosa da evidência científica. Rev. bras. Fisioter, 11(1), 8389.

Silva, D. S. D. et al. (2015). Depressão e risco de suicídio entre profissionais de Enfermagem: revisão integrativa. Revista da Escola de Enfermagem da USP, 49(6), 1023-1031.

Sousa, C. H. P., \& Tavares, C. M. M. (2020). Depressão e suicídio nos discentes de enfermagem e o conhecimento docente sobre este sofrimento. Research, Society and Development, 9(6), (e39963434).

Souza, C., \& Moreira, V. (2018). Tristeza, depressão e suicídio melancólico: a relação com o Outro. Arquivos Brasileiros de Psicologia, 70(2), 173-185.

Trujillo, A. H., Elías I. E. E., \& Acosta L. Y. M. L. (2013). Factores de riesgo relacionados con la conducta suicida en la infancia y adolescencia. MEDISAN, 17(12), 9027-9035. 